\title{
Hepatitis foundation international biosimilars issue brief
}

\begin{abstract}
Biosimilars are biological medical products highly similar to the original products manufactured by different pharmaceutical companies. Biosimilars inherit a critical role that addresses the growing need for mechanism-based drugs needed to treat diseases such as hepatitis, HIV and various cancers. In this issue brief, the developmental background, approval process, regulatory review and safety procedures and naming process will be discussed in detail. Benefits and cost to the patient population will also be examined, contrasting how biosimilars can be interchanged or substituted with other drugs to produce near identical health outcomes at affordable prices. Although biosimilar development appears promising, guidelines to proper labeling and surveillance of adverse effects are still being conducted. Patients tend to respond differently to drug therapies, making biosimilars the patient-specific treatment alternative. The Food and Drug Administration (FDA) continues to expand on its recommendations to improve regulation, safety precautions and transparency across all levels of health care for medical providers and their patients.
\end{abstract}

Volume 2 Issue 3 - 2016

\author{
Ivonne Fuller Cameron, Lauren Healy, Willis \\ Chang \\ Hepatitis Foundation International, USA
}

Correspondence: Ivonne Fuller Cameron, President \& CEO, Hepatitis Foundation International, World Headquarters, 812 I Georgia Avenue, Suite 350, Silver Spring, MD 20910, USA, Tel 3015659410, Email ifcameron@hepatitisfoundation.orgg

Received: November 19,2015 | Published: August II, 2016

Keywords: hepatitis, biosimilars, cancer, diabetes, HIV, neupogen

Abbreviations: FDA, food and drug administration; BPCIA, biologics pricing competition and innovation act; HFI, hepatitis foundation international; WHO, world health organization; INN, international nonproprietary name; EMA, european medicines agency; EC, european commission; HB319, house bill 319; USAN, united states assigned name

\section{Introduction}

Biologics are medicines that have revolutionized the treatment of many serious and life threatening diseases, including hepatitis. The introduction of biologics was timed to address the growing need for mechanism-based drug therapies for diseases such as hepatitis, HIV and cancer. ${ }^{1}$ These medicines are composed of very large and complex molecules (i.e. proteins and enzymes) that are produced from a living organism or its products. Biosimilars are medications deemed highly similar, but not identical to, an already-approved medicine. Biosimilars are both structurally and clinically alike in terms of safety, potency and purity to medications already licensed by the US Food and Drug Administration.

The Food and Drug Administration (FDA) has faced challenges in the development of guidelines for biosimilar versions of biotech therapies. ${ }^{2}$ It has been a timely and costly effort for companies to develop a follow-on biotech therapy. Biosimilars can be identified as "biopharmaceuticals similar in composition to the original product, but not necessarily interchangeable". ${ }^{3}$ Because biologics are made using living cells, copies will only be related, not identical to the original product. Because of the size and complexity of the molecules that make up these medicines, it is currently not possible to know exactly how biosimilars differ from the original product and whether these differences matter. The FDA has been granted authority through the Biologics Pricing Competition and Innovation Act (BPCIA), a provision of the Affordable Care Act, to create an accelerated approval process for biosimilar products.

The Hepatitis Foundation International (HFI) has developed this issue brief to define biosimilars, provide an overview, examine the barriers to implementation and review policy considerations related to their adoption and implementation. HFI is a 501(c) (3) non-profit organization established in 1994 to eradicate viral hepatitis for 400million people globally. Section 501(c) (3) is the portion of the US Internal Revenue Code that allows for federal tax exemption of nonprofit organizations, commonly known as "charity organizations". HFI is also dedicated to increasing and promoting health and wellness, as well as, reducing the incidence of preventable liver-related chronic diseases and lifestyles that negatively impact the liver. Some of these diseases include obesity, diabetes, hepatitis, substance abuse, HIV/AIDS, cardiovascular disease, fatty liver and liver cancer. HFI implements its mission through our touchstones to educate, prevent, serve and support and reach well over 5million patients and health care professionals annually (Table 1) (Figure 1).

\section{Biosimilars}

Biosimilars are related to their biologic counterparts and are approved for therapeutic, preventative and diagnostic purposes. They are produced in a similar manner with the expectation of creating a low-cost, high-quality alternative to the original biologics when the patent terms of the reference biologic expire. ${ }^{4}$ Major differences between traditional pharmaceuticals and biosimilar therapies include the size, complexity and sensitivity of the molecules, all of which have implications for manufacturing and regulating copies of these medicines.

Biosimilar manufacturers must "demonstrate similarity of the drug's efficacy and safety". ${ }^{5}$ With approximately eighteen biosimilar products approved in Europe thus far, biosimilars are now becoming a treatment option in the United States. On March 23, 2010, the United States Congress enacted the Biologics Price Competition and Innovation Act (BPCIA) as a means to improve patient accessibility to innovative medical therapies. ${ }^{6}$ The BPCIA authorized the FDA to regulate biosimilar drug products acting as an amendment to the Public Health Service Act. This amendment created an abbreviated 
licensure pathway for biological products that are considered "biosimilar" to or "interchangeable" with FDA-licensed biological products. ${ }^{2}$ With this amendment, the FDA ensures that biosimilars will have no meaningful clinical differences, in terms of safety and efficacy, in comparison to the reference product ${ }^{5}$ although that does not mean the products will be identical and just like with reference drugs, patients may not respond to treatment as expected and require a different course of treatment.

The BPCIA created an opening to increase availability of biosimilar products to patients who face financial challenges to purchasing expensive biological products. Recently, biosimilars have gained attention in the U.S. due to the increasing need for affordable health care treatments and the looming threat of the "patent cliff" for many biologics currently in Calo Fernández B.?

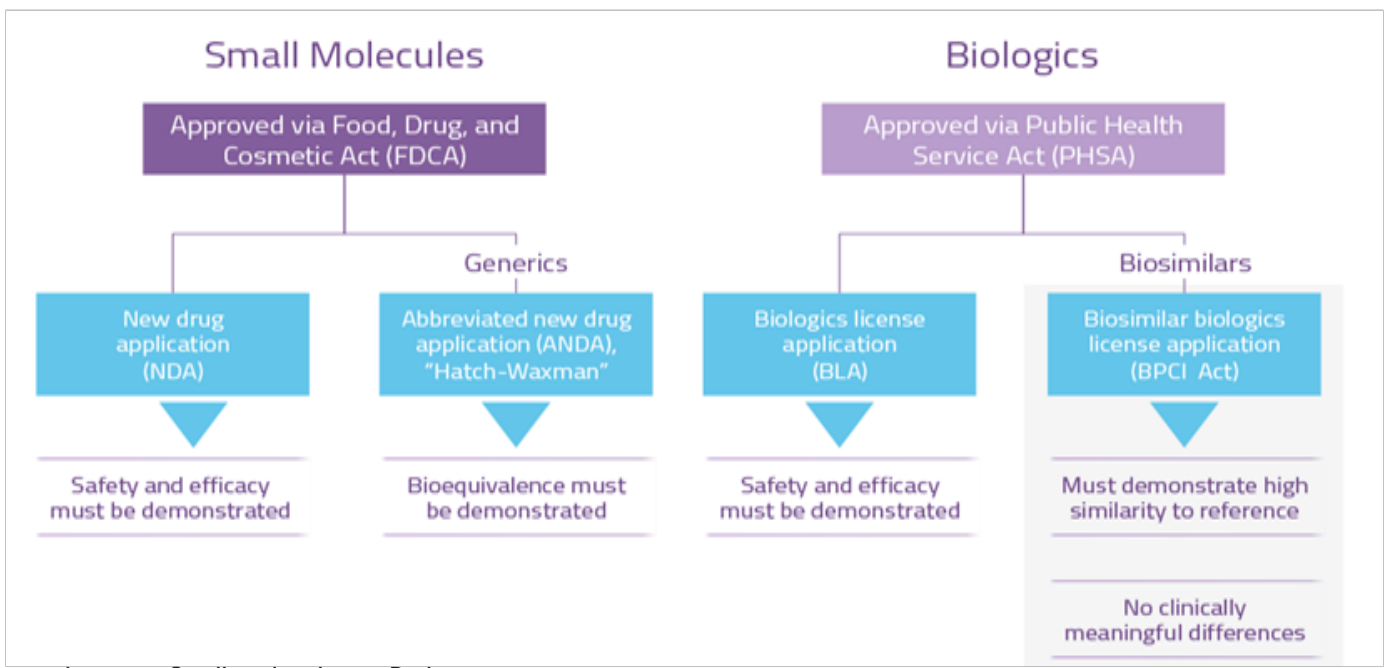

Figure I Comparison between Small molecules vs. Biologics

Table I The major differences between traditional pharmaceuticals and biological therapies

\begin{tabular}{|c|c|c|}
\hline \multicolumn{3}{|c|}{ Drug comparison: small vs. large molecules } \\
\hline & Small, therapeutic molecules(Drugs) & Biological therapeutic molecules \\
\hline \multirow{3}{*}{ Size of molecule } & -Small (Single Molecule) & -Large (Mixture of related molecules) \\
\hline & & \\
\hline & -Low molecular weight & -High molecular weight \\
\hline Complexity of molecule & $\begin{array}{l}\text { Drugs generally have well defined chemical } \\
\text { structures, and a finished drug can usually be } \\
\text { analysed to determine all its various components. }\end{array}$ & $\begin{array}{l}\text { It is difficult, and sometimes impossible, to } \\
\text { characterize a complex biologic by testing } \\
\text { available in the laboratory, and some of the } \\
\text { components of a finished biologic may be } \\
\text { unknown. }\end{array}$ \\
\hline Source/Origin & Chemicals & $\begin{array}{l}\text { Living organisms to include plant or animal cells, } \\
\text { micro organisms, biological fluids (e.g. plasma). }\end{array}$ \\
\hline Manufacturing processes & $\begin{array}{l}\text { Typically manufactured through chemical synthesis } \\
\text { by combining specific chemicals in a controlled } \\
\text { environment in an ordered process producing an } \\
\text { extremely pure product. }\end{array}$ & $\begin{array}{l}\text { Typically manufactured in biological systems such } \\
\text { as micro organisms, plant or animal cells. Most } \\
\text { biologics are very large, complex molecules or } \\
\text { mixtures of molecules of acceptable purity. Many } \\
\text { biologics are produced using recombinant DNA } \\
\text { technology. }\end{array}$ \\
\hline Characterization & $\begin{array}{l}\text { Easy to characterize completely using standard } \\
\text { analytical assays. }\end{array}$ & $\begin{array}{l}\text { Cannot be characterized completely due } \\
\text { to the complex molecular composition and } \\
\text { heterogeneity. }\end{array}$ \\
\hline Stability & Usually stable at room temperature & $\begin{array}{l}\text { Usually unstable at room temperature } \\
\text { necessitating storage under refrigerated } \\
\text { conditions. }\end{array}$ \\
\hline Immunogenicity & Usually non immunogenic & Usually immunogenic \\
\hline
\end{tabular}

Source: Shoemaker 
Shortly after the FDA finalized its first guidance on the biosimilar drug approval process, it authorized its first biosimilar, Sandoz's Zarxio. This biosimilar is based off of the reference drug, brand-name filgrastim, marketed as Neupogen (Amgen), which was originally approved in 1991 for many clinical uses including aiding recovery from neutropenia in cancer patients undergoing chemotherapy. Neupogen had total sales topping more than \$1billion in 2014. This first biosimilar was granted full indication extrapolation, meaning that the drug does not need to be tested for each medical condition in order to be approved, if the biologic was approved for these medical conditions. Much of the FDA's biosimilar guidance, however, has indicated that biosimilars will be evaluated on a case-by-case basis.

\section{Abbreviated regulatory review and safety}

Biosimilars are examined using a modified regulatory review process based on a comparison to the product that has already been approved rather than demonstrating safety and efficacy directly. Biosimilars are required to be highly similar to and have no clinically meaningful differences with the biologics they copy. However, even minor changes in the raw materials or the manufacturing process (introduced due to the inherent differences in biological systems) could cause the copy to deviate from the original product. Subsequently, the review process currently includes testing to ensure safety.

In addition, long-term post-market surveillance will be essential for all biologics, including biosimilars. Both physicians and patients will need to be educated about the nature of biologics to ensure these products are used as intended. Biosimilars are not like generic drugs and cannot be assumed to be appropriate for substitution or for switching products during a course of therapy.

\section{Extrapolation}

Extrapolation is the process of extending conclusions from studies on one medical product populations to make inferences about a similar medical product. FDA Draft Guidance for Industry states that "extrapolation of indications for a biosimilar is possible given robust evidence and scientific justification." FDA provided guidance on extrapolation of approval across multiple clinical indications for biosimilars. ${ }^{8}$ Extrapolation is justified based on similar mechanisms of action, target/receptor interactions and molecular signaling; product structure interactions with the target or receptor; PK, expected toxicities and information based on mechanism of action. Indication extrapolation occurs when the regulatory bodies apply approval for biosimilars to have one medical use to an additional use that mirrors another of the biologic reference product's approved indications. Extrapolation does not require the rigorous clinical testing that is typical for drugs to obtain approval for specific indications. Biosimilar manufacturers are required to provide evidence of "therapeutic similarity" for use with the disease or patient population in question. If the relevant mechanism of action of the active substance and the target receptor(s) involved in the biologic and in the biosimilar are the same, extrapolation is usually not problematic. However, when a biologic or a biosimilar have multiple receptors and a complex mode of action, extrapolation can be less accurate.

Extrapolation provides a clear shortcut for biosimilar approval, requiring far less clinical testing compared to the approval process of the biologic. Reducing the need for vast clinical testing results in a high profit for the manufacturer and a lower cost of treatment for the payers and the patients. Requiring extensive testing could raise the cost to manufacture, in turn, raising the cost for the patient.

\section{Naming}

All medicines are assigned a non-proprietary name by which they can be identified. The naming conventions for biosimilars are still under development. In August 2015, FDA issued draft guidance and proposed a framework for naming all biologics, including biosimilars. This proposal would assign a distinguishing suffix to all biologics, which would be attached to the name by a hyphen. This approach enables physicians to prescribe with specificity and helps ensure that products are dispensed according to the intent of the physician. Also important, the suffix is purposed to accurately facilitate associations of various drug problems with the correct medicine. Because copies of biologics will not be identical to the original product, the differences may be important for some patients. It is critical for doctors to know which product the patient is reacting to Walsh. By convention, small molecular drugs and their generic formats share a nonproprietary name. The World Health Organization (WHO) assigns an International Nonproprietary Name (INN). Like many countries, the United States considers the INN but has its own naming conventions and issues each molecule a "USAN"-United States Assigned Name. These allow physicians to identify the chemical composition to improve prescribing methods for patients. ${ }^{5}$ It is important that guidelines for biosimilars are in place, because biosimilars are not structurally identical to the product they copy and those differences may matter to some patients.

Naming is an important aspect of drug development. It includes a tracking process linking adverse events and/or successes from clinical trials and post-surveillance observations. Given the vast differences between chemical compounds and biologics, biosimilars need to be given distinguishable non-proprietary names. By providing this information dating back to the point of prescription, distinguishable names facilitate tracking a biosimilars success and/or failure. By tracking the biosimilar, it is possible to effectively determine the cause of an adverse event by creating a direct route back to the origin of the problem. Issues that may arise for a specific biological product can be untraceable without distinguishable names. A distinguishable naming system is an important component of improving transparency in the health care arena.

For this reason, HFI, along with other patient biologic safety and access forums, has requested the FDA and the World Health Organization (WHO) consider guidelines for distinguishable nonproprietary naming conventions in order to better track adverse events and ensure the safety of patients relying on biosimilars. The European Medicines Agency (EMA) has successfully adopted the distinguishable non-proprietary naming system. ${ }^{9}$ The EMA allows biosimilar products to use identical non-proprietary names as the reference product. However, in 2012 the European Commission (EC) mandated all European Union member states to label all biological medications by product name and batch number. ${ }^{10}$ Consensus on how biosimilars are to be labeled is in process as the FDA recently released its 2016 labeling for Biosimilar Products guidance.

\section{Cost and patient benefit}

Biosimilars, along with their respective biologics, are expected to increase the treatment choices available to patients and may be less costly for some patients. However, biologics-both innovative and biosimilar medicines are expensive to manufacture.

The price of biosimilars is not expected to follow the generic drug cost structure, which can see a reduction of $90 \%$ in a matter of 
weeks. ${ }^{4}$ In the generic drug arena, when a reference product patent expires, revenue will decline in a manner as if falling from a cliff, hence the term patent cliff..$^{11}$ However, this is only true for smallmolecule drugs. The FDA's approach for regulating small-molecule generic drugs cannot be applied to biologics because biologics are complex molecules manufactured in living systems. ${ }^{12}$ This leaves competing firms open to manufacturing the biosimilar product at a cheaper cost, thereby making it more affordable by giving patients more options of using either a biologic or a biosimilar product. Currently, biologics typically do not face generic competition after their original patent protection has expired, thus maintaining the high drug prices indefinitely. It is the lack of generic competition in biologics that leads Rand Corporation to estimate that biosimilars will lead to a $\$ 44.2$ billion reduction in direct spending on biologic drugs from 2014-2014, which adds up to about 4percent of total biologic spending over the same time frame. The FDA has made it clear that biosimilars and traditional generic drugs will be regulated differently. Biosimilars require at minimum one head-to-head clinical trial to confirm similarity with the reference drug, and not all biosimilars will be deemed "interchangeable". Potential competition may result from the introduction of biosimilars to the market, however, the magnitude is unknown and how the savings will be shared among payers, patients, providers and tax payers is unclear (Figure 2).

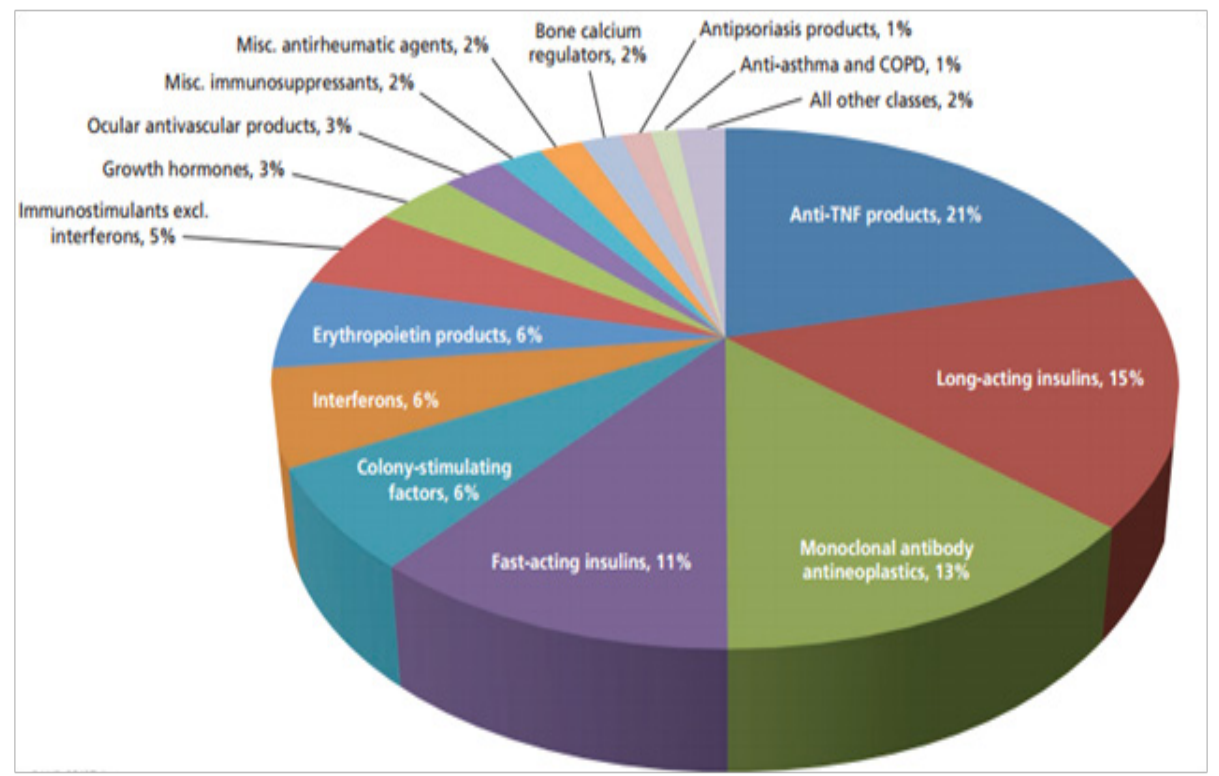

Figure 2 Potential cost savings across biologic classes. Pie Chart compiled by Rand Corporation using data on more than I00 biologics, including all blockbuster biologics with sales over $\$ \mathbf{I}$ billion and many products with smaller markets to estimate the potential direct cost savings from biosimilars. ${ }^{13}$

Inflectra $^{T M}$ (Infliximab), a biosimilar recently approved in Europe and Canada, is a chimeric monoclonal antibody drug designed to target arthritis and Crohn's disease. Inflectra ${ }^{T M}$ is selling 45percent less than the original biologic, Remicade, proving that affordable biosimilars are possible. ${ }^{4}$ This recent EU biosimilar experience suggests that there may be differences in price, depending on their position in the market. This is beneficial to patients, as it increases the variety of treatments for patients. It is of the utmost responsibility of biosimilar manufacturers and service providers to keep patient safety and accessibility to a top priority in making decisions that impact patient healthcare.

Subsequently, biologics have revolutionized patient care. For some patients, biologics are the best method (and option) of treatment to improve quality of life. However, biologics can vary in success from patient to patient. A medication's success depends on the way it interacts with each individual's biological makeup. Since it may take up to six months for a biologic to begin working, it is recommended that patients on biologics should have their health continuously monitored to check for any adverse effects. ${ }^{13}$

\section{Substitution and switching}

Biosimilars will be highly similar, but not identical to the product they intend to copy. The US law defines two levels of approval by FDA-biosimilar and interchangeable. A biosimilar product is defined as one that is "highly similar" to the reference product, with only minor differences in clinically inactive components. There are no clinically meaningful differences between the biosimilar and the reference product in terms of safety, purity and potency. An interchangeable biological product is one found to be biosimilar, which means it is expected to produce the same clinical result as the reference drug in any given patient. The risk in terms of safety or diminished effectiveness of alternating or switching between use of the product and the reference is no greater than the risk of using the pioneer without any alteration or switch.

A product approved by FDA as biosimilar will be highly similar and therefore as safe and effective as the product it has completed clinical trials with. However, a biosimilar cannot be considered appropriate for repeated switching with the original product unless it has undergone clinical trials to treat the specific disease it is being substituted for.

A biosimilar approved as interchangeable will have demonstrated to have the same effect in any given patient and that the patient will be at no greater risk of adverse events or an unwanted immune response as a result of switching between the original and the biosimilar. Current thinking among physicians and patient advocacy groups shows that biosimilars should be dispensed based on prescription recommendations from the physician's office. Only those medicines designated as "interchangeable" are appropriate for substitution by 
the pharmacist, without the physician's involvement. The physician and pharmacist team should have a common understanding of what the patient received.

Although FDA approves drugs as biosimilar or interchangeable, state law governs when a pharmacist can substitute one product for another. A number of states have adopted laws permitting substitution of interchangeable products if certain conditions are met. Most noteworthy of these are that the prescriber(s) can specify that the product should be dispensed as written, and substitution is not permitted. Additionally, bills generally require that the pharmacist informs the prescriber(s) of the product received by the patient.

Louisiana House Bill 319 (HB319) emphasizes the importance of transparency, ensuring that patients know whether they are taking a biologic or biosimilar. Louisiana Governor, Bobby Jindal, signed the bill into law on July 1,2014 requiring pharmacists to notify prescribers within five days if and when a biologic drug is substituted. ${ }^{14}$ It is critical for all members of a patient's healthcare team to know what medications their patient is taking. Anytime treatment is altered, the medical professionals involved should be notified. It is critical to the patient's health and well-being that they have a transparent and trusting relationship with their healthcare providers. HB319 seeks to rectify this for patients.

\section{Labeling}

When the FDA approved the first biosimilar, Zarxio ${ }^{\mathrm{TM}}$, the label that was approved was essentially identical to the label of the reference biologic. The label fails to clearly state that Zarxio is a biosimilar and that is has not been evaluated for substitution and extrapolation. It is not made clear on the label that the information being shown is for the biologic, not the biosimilar.

For full prescribing information, FDA recommends that biosimilar product labeling incorporates relevant data and information from the FDA-approved labeling for the reference product, along with any and all modifications specific to the biosimilar product. A biosimilar product is not required to have the same labeling as its reference product. The FDA recommends that biosimilar labeling include biosimilar product-specific data necessary to regulate safe and effective use. However, they do not recommend that comparative data supporting the demonstration of bio similarity be included in biosimilar product labeling, meaning they do not recommend that it be explicitly stated that the drug is a biosimilar. This approach is in an effort to avoid potential confusion or misinterpretation of the comparative data, according to Leah Christl, Associate Director for Therapeutic Biologics and lead of the Therapeutic Biologics and Biosimilars Staff in the Office of New Drugs.

\section{Adverse event surveillance}

Government regulatory policies of biosimilars will evolve based on the actual experiences derived from the patient population. This calls for a closer examination and monitoring of adverse events reported from clinical trials and post-market surveillance tracking data. In the U.S, biosimilars are typically prescribed by a physician. ${ }^{2}$ However, this does not hold true everywhere. A pharmacist from another county could substitute a biosimilar in place of a biologic without documenting any adverse effects. Certain biosimilars are also not regulated and freely available in other countries. With increasing globalization of manufacturing and testing, it becomes necessary to unify efforts in rendering proper advice to patients globally. It would be necessary to create a global surveillance system with cooperation from regulators, patient advocacy groups and health care providers worldwide for making safer, more accessible and affordable care for everyone.

\section{Acknowledgements}

Sincere gratitude is expressed for the following individuals for their contributions on the Biosimilars Issue Brief: Ivonne Cameron, Lauren Healy and Willis Chang.

\section{Conflict of interest}

Author declares that there is no conflict of interest.

\section{References}

1. Boutwell AE, Allen SA, Rich JD. Opportunities to address the Hepatitis C epidemic in the correctional setting. Clin Infect Dis. 2005;40(Supp 5):S367-S372.

2. Jill Wechsler. Development of biosimilars creates challenges for FDA, manufacturers. Formulary Journal. USA; 2011.

3. Gottlieb S. Biosimilars: Policy, clinical, and regulatory considerations. Am J Health Syst Pharm. 2008;65(14 Suppl 6):S2-S8.

4. Peter J Pitts, Roger Stark. Biosimilars: The precarious struggle between cost-driven health care policy and patient-controlled care. Washington Policy Centre, Washington, USA; 2012.

5. Shoemaker, David. The U.S. biosimilar pathway: policy precedes science. Applied Clinical Trials. 2015;24(6/7):40-45.

6. Strand V, Cronstein B. Biosimilars: how similar? Intern Med J. 2014;44(3):218-223.

7. Calo Fernández B, Martínez Hurtado JL. Biosimilars: Company strategies to capture value from the biologics market. Pharmaceuticals ( $\mathrm{Ba}$ sel). 2015;5(12):1393-1408.

8. Tom DiLenge, Sara Radcliffe. Biotechnology Industry Organization, Washington, USA; 2014.

9. Grabowski HG, Kyle M, Mortimer R, et al. Evolving brand- name and generic drug competition may warrant of revision of the Hatch-Waxman Act. Health Aff (Millwood). 2011;30(11):2157-2166.

10. Mahn TG, Dhavan GM. Biosimilars vs. Generics Major Differences in the Regulatory Model. Pharmaceutical Compliance Monitor, Texas, USA; 2012.

11. Mary Rice. EMEA takles "generic" biologic drug issues. JNCI J Natl Cancer Inst. 2006;98(7):435-436.

12. Labeling for Biosimilar Products Guidance for Industry. Food and Drug Administration, Rockville, Washington, USA;

13. Frank RG. Regulations of follow-on biologics. $N$ Engl J Med. 2007;357:841-843.

14. Anonymous. World Preview 2018. Embracing the Patent Cliff. Evaluate Pharma, USA; 2012. 